\title{
THE INFLUENCE OF NON-MAGNETIC SUBSTRATE ON SPATIAL DISTRIBUTION OF GAUSSIAN FLUCTUATIONS OF MOLECULAR FIELD IN MAGNETIC NANOPARTICLES AND ULTRATHIN FILMS
}

\author{
R. ŁosIŃSKI* AND Z. ONYSZKIEWICZ \\ Institute of Physics, A. Mickiewicz University \\ Umultowska 85, 61-614 Poznań, Poland
}

\begin{abstract}
An Ising $(s=1)$ model of ferromagnetic nanoparticles and ultrathin films of the sc structure deposited on a non-magnetic substrate is considered. The substrate was assumed to affect the crystal field around the atoms lying closest to it. Consequently, the one-ion anisotropy constant of spin moments of these atoms becomes dependent on the appropriate component of the tensor of the magnetic structure deformation. This dependence was assumed to be linear. To obtain approximations of the Gaussian fluctuations of molecular field, the generalised equilibrium reduced density operator, along with the Feynman diagram technique were used. As a result, temperature dependences of the spatial distributions of mean fluctuations of the magnetic field versus the changes in the one-ion anisotropy constant induced by the non-magnetic substrate were obtained.
\end{abstract}

PACS numbers: $75.10 . \mathrm{Hk}, 75.70 .-\mathrm{i}$

This paper aims at presenting the possibly most accurate calculations of the spatial distribution of the mean fluctuations of the molecular field $\delta y_{f r}$ for a simple model of magnetic nanoparticles and ultrathin films for various surface one-ion anisotropies describing the interaction with non-magnetic substrate and for different temperatures $T$. We consider nanoparticles and ultrathin films whose ferromagnetically ordered spins are divided into $R$ monoatomic layers parallel with the surface of the substrate. The position of each atom is given by the number of the layer $r=1,2, \ldots, R$ and the bidimensional vector $f$. Our considerations are restricted to the nearest neighbour interactions only. Finally, let us assume that the magnetic properties of our magnetic systems are satisfactorily described by a simple cubic Ising model $H_{m}$ with one-ion anisotropy $(s=1)$.

\footnotetext{
*e-mail: rlosin@amu.edu.pl
} 
Usually, when investigating theoretically macroscopic bulk systems we do not have to take into account their interaction with the environment regarding the short-range character of intermolecular interactions. An effective interaction among the molecules of the system and its environment could only occur through the atoms on the systems surface or near it. The number of interacting atoms usually makes an insignificant part of all atoms of the system. However, considering ultrathin solid films and similar systems, the number of atoms interacting with the environment is frequently of the same order of magnitude as the total number of atoms in the system. Moreover, nanoparticles or ultrathin solid films are deposited on bulk substrates whose structure influences that of the films. Because of that, we have to take account of the interaction between the substrate and the nanoparticles or ultrathin film. Usually, in order to do that we introduce phenomenological surface parameters. The values of these parameters significantly influence the physical properties of thin solid films which is for example evidenced in their spectra of collective excitations.

Therefore we assume that our ferromagnetic nanoparticle or ultrathin film is deposited on a bulk substrate which can be described by a Hamiltonian $H_{p}$. In this case we can use the generalised reduced density operator [1]

$$
d_{m}=\exp \left[\beta\left(F_{m}-H_{m}-H_{m}^{\prime}\right)\right],
$$

where

$$
H_{m}^{\prime}(\beta)=\operatorname{Tr}_{p}\left[H_{m p} \exp \left(\beta\left(F_{p}-H_{p}\right)\right)\right]=\left\langle H_{m p}\right\rangle_{p},
$$

$H_{m p}$ is the term describing the interaction between the nanoparticle or the film and the substrate, $\operatorname{Tr}_{p}[\ldots]$ stands for a partial trace over the Hilbert space of the substrate with Hamiltonian $H_{p}$, and $\beta=\left(k_{\mathrm{B}} T\right)^{-1}$.

Let us assume that $H_{m p}$ has an ordinary bilinear form

$$
H_{m p}=\sum_{f} A_{f} P_{f}\left(S_{f}^{z}\right)^{2},
$$

where $P_{f}$ is a one-particle operator of the substrate and $A_{f}$ is a particle(film)substrate interaction coefficient.

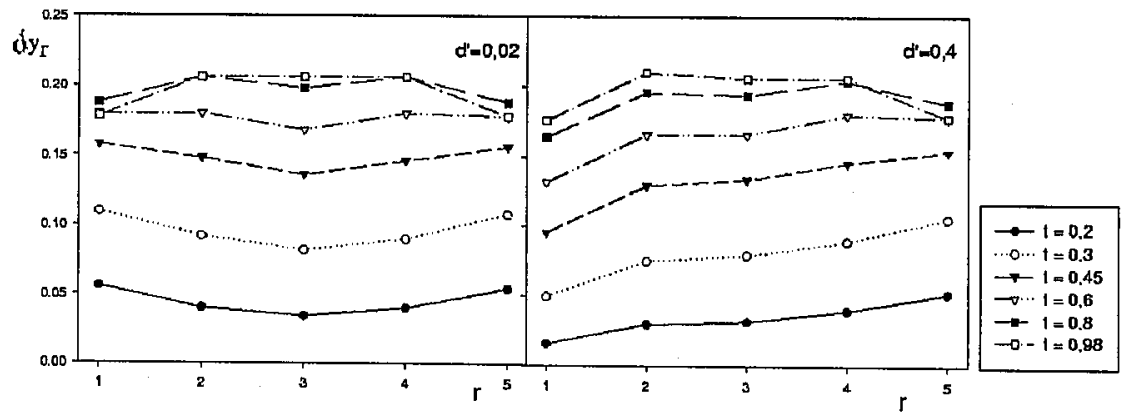

Fig. 1. Spatial distribution of the mean fluctuations of the molecular field $\delta y_{r}=$ constant $(f)$ (related to the mean molecular field value) in cubic ferromagnetic ultrathin film for different temperatures $t$ and different coefficients $d^{\prime} ; C^{\prime}=0.25$. 

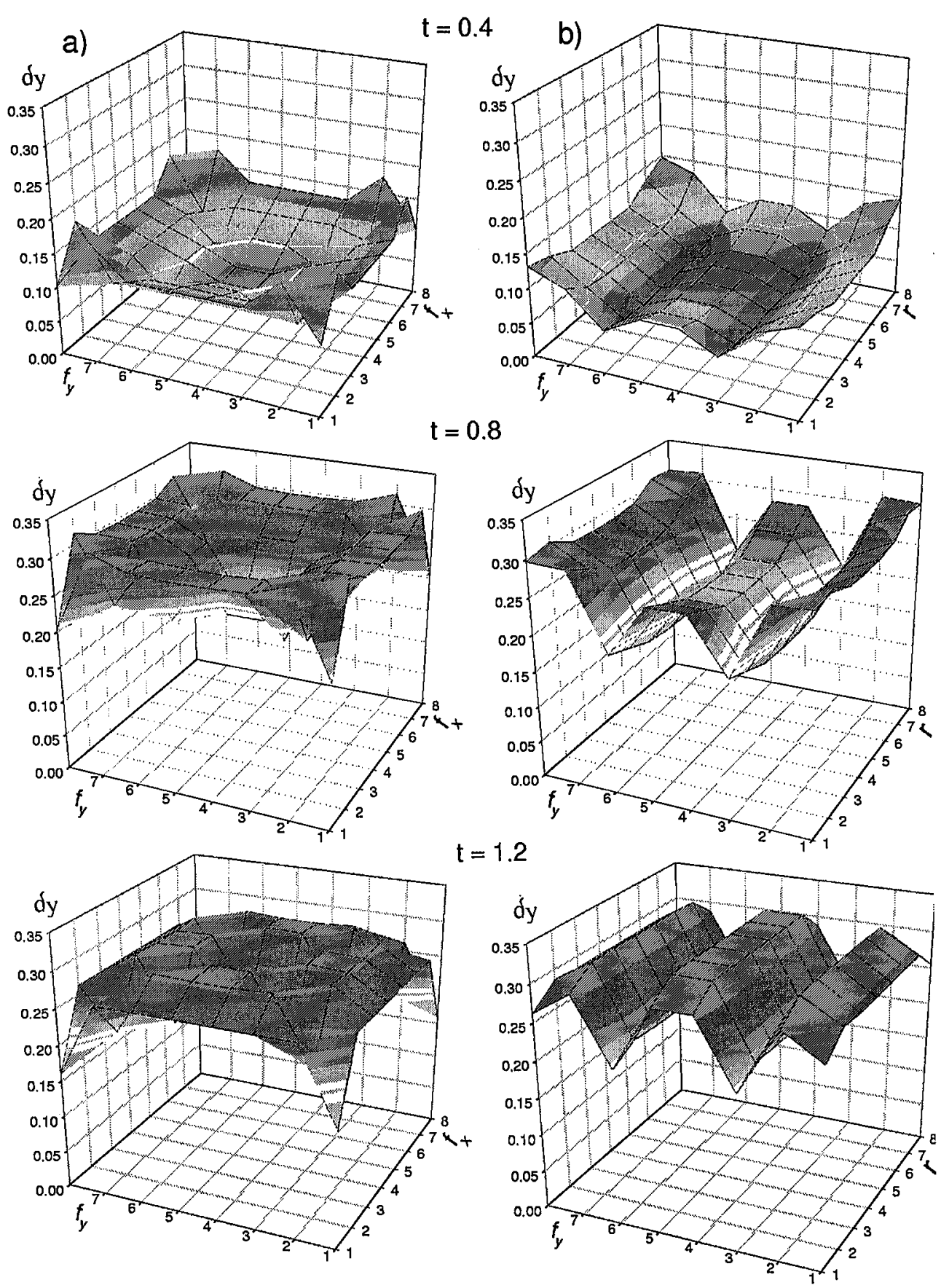

Fig. 2. Spatial distribution of the mean fluctuations of molecular field $\delta y_{f r}$ in ferromagnetic nanoparticle of the size $8 \times 8 \times 8$ spins for different temperatures $t$ and for $d^{\prime}=0.3, C^{\prime}=2.5:(\mathrm{a})$ in the plane $r=1,(\mathrm{~b})$ in the wall $3 . f_{x}$ and $f_{y}$ are the coordinates of the vector $f$. 
Now, we assume that $A_{f}\left\langle P_{f}\right\rangle_{p}$ is a linear function of the $z$ component of the tensor of the magnetic structure deformation $\epsilon$. On the basis of Eqs. (1)-(3) and our assumptions, the effective Hamiltonian is given by (for $s=1$ )

$$
H_{m}+H_{m}^{\prime}=-\frac{1}{2} I \sum_{f r} \sum_{f r^{\prime}} S_{f r}^{z} S_{f r^{\prime}}^{z}-\sum_{f r} D_{r}\left(S_{f r}^{z}\right)^{2}+\frac{1}{2} C \epsilon^{2},
$$

where the effective one-ion anisotropy constants

$$
D_{r}= \begin{cases}D^{\prime \prime}+D^{\prime} \epsilon & \text { for } r=1 \\ D & \text { for } r=2,3, \ldots, R-1, \\ D^{\prime \prime} & \text { for } r=R\end{cases}
$$

and the last term in (4) represents classical elastic energy of the ferromagnetic system.

In order to obtain the spatial distribution of the mean fluctuations of molecular field $\delta y_{f r}$ we report to the generalized equilibrium density operator (1) and the Gaussian fluctuation approximation (GFA), which is a modified version of the high density expansion method and has been proposed in $[2,3]$. GFA is an improvement of the molecular field approximation (MFA) due to the self-consistent inclusion of Gaussian fluctuations of this field. The essential new element of GFA is the summation of the Feynman diagrams of the same structure of recurrent formulae at each stage of the calculations. Owing to this procedure the theory becomes internally consistent and does not lead to non-physical results such as, for example, complex Curie temperature (see [4]).

Appropriate equations in GFA are solved numerically for the parameters: $d=$ $D / 4 I=1, d^{\prime \prime}=D^{\prime \prime} / 4 I=0.8, C^{\prime}=C / 4 N I=0.25$ or $C^{\prime}=2.5(N$ - number of spins in one monoatomic layer), and the results are presented graphically. Figure 1 presents the spatial distribution of the mean fluctuations of the molecular field $\delta y_{r}$ (in relative units) for different temperatures $t=k_{\mathrm{B}} T / 4 I$ and for different film-substrate interaction coefficient $d^{\prime}=D^{\prime} / 4 I$. Spatial distribution of the mean fluctuations of the molecular field $\delta y_{f r}$ in cubic ferromagnetic nanoparticle of the size $8 \times 8 \times 8$ for different temperatures $t$ and for $d^{\prime}=0.3$ are shown in Fig. 2 .

In conclusion, we can say that the results in Figs. 1 and 2 prove that spatial distribution of the mean fluctuations of the molecular field $\delta y_{f r}$ in ferromagnetic nanoparticles and ultrathin films may, to a significant degree, depend on the value of the $d^{\prime}$ coefficient characterizing the influence of the non-magnetic substrate on both of them. The results obtained seem valuable to us as they can be experimentally verified by the measurements $\delta y_{f r}$ applying the technique based on the Mössbauer effect.

This work is supported by the Committee for Scientific Research under grant No. 2P03B075 14.

\section{References}

[1] Z. Onyszkiewicz, Phys. Lett. A 163, 450 (1992).

[2] Z. Onyszkiewicz, Phys. Lett. A 76, 411 (1980).

[3] Z. Onyszkiewicz, Physica A 103, 257 (1980).

[4] Z. Onyszkiewicz, A. Wierzbicki, Phys. Lett. A 116, 335 (1986). 